\title{
Designing an Integrated All-Optical Analog to Digital Converter
}

\author{
Sajjad Moshfe ${ }^{a}$, Mohammad Kazem Moravvej-Farshi ${ }^{\text {b,* }}$, Kambiz Abedi ${ }^{\mathrm{c}}$ \\ ${ }^{a}$ Department of Electrical Engineering, Science and research branch, Islamic Azad University, \\ Tehran, Iran

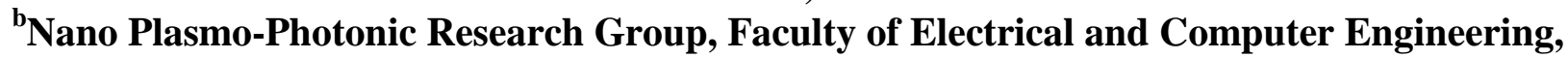 \\ Tarbiat Modares University, Tehran, Iran \\ ${ }^{c}$ Department of Electrical and Computer Engineering, Shahid Beheshti University, Tehran, \\ Iran
}

Corresponding author Email: moravvej@modares.ac.ir

Regular paper: Received: Jun. 16, 2019, Revised: Oct. 2, 2019, Accepted: Nov. 2, 2019,
Available Online: June. 30, 2020, DOI: 10.29252/ijop.14.1.3

\begin{abstract}
We present the procedure for designing a high speed and low power alloptical analog to digital converter (AO-ADC), by integrating InGaAsP semiconductor optical amplifier (SOA) with InP based photonic crystal (PhC) drop filters. The self-phase modulation in the SOA can shift the frequency of the Gaussian input pulse. The two output PhC based drop filters are designed to appropriately code the frequency-shifted analog signals by the SOA, converting them to four desired digital output levels. Our numerical results show that in an appropriately designed AO-ADC, the center wavelength (1572 nm) of an amplitude modulated Gaussian pulse of $1.8 \mathrm{ps}$ width and $1.56 \mathrm{pJ}$ energy can be shifted by $6.7 \mathrm{~nm}$, by the SOA, and then be quantized and coded to four digital levels (00, 01, 10, and 11). The two point-defect $\mathrm{PhC}$ drop filters, compensating the effect of the frequency shift by SOA, minimize the AO-ADC integral and differential nonlinearity errors.
\end{abstract}

KEYWORDS: Semiconductor Optical Amplifier (SOA), Self-Phase Modulation (SPM), Photonic Crystal (PhC), Analog to Digital Converter (ADC).

\section{I.INTRODUCTION}

Continuous nature of the real world signals makes an analog to digital converter (ADC) an inseparable part of any digital process. An electronic ADC faces with a few challenging issues, such as the jitter of the sampling window and the ambiguity of the comparator. To overcome these issues, researchers have proposed semi-optical ADC $[1,2]$. Moreover, various all-optical ADCs (AO-ADCs) have also been proposed in this regard [3-20]. An AO-ADC consists of a mode-locked laser, generating high speed pulses with ultra-low jitters, as the sampling part, an optical quantizer, and an optical coder. The optical ADC designers have substituted the latter two optical parts for the electronic parts in a semioptical ADC to enhance its conversion speed [3].

The Kerr effect has been rigorously used in the design of the AO-ADCs. In the early '90s, a 3bit AO-ADC was developed experimentally, using the cross-phase modulation of a 200-m single mode fiber loop employed as a nonlinear interferometer [4]. A decade later, a 4-bit ADC based on hard limiters was presented [5]. The hard limiters consist of alternating layers of materials having different Kerr nonlinearities. Other techniques such as self-frequency shifting [6, 7], slicing supercontinuum spectrum [8], and self-phase modulation (SPM) [9] in a long linear or nonlinear fiber were also used to design AOADCs. Later, employing the self-frequency 
shift and SPM induced spectral compression in a highly nonlinear fiber, a 4-bit ADC operating at $100 \mathrm{~Gb} / \mathrm{s}$ were designed [10]. They employed the self-frequency shifting to quantize the analog signal and used the spectral compression to improve the resolution. Nevertheless, all these fiber-based ADCs, reported so far, have exploited long fibers that require high input power that makes it unsuitable for integration. Following the same trend, by using the multistage approach for SPM-based spectral compression in fiber and improving the spectral compression ratio more than twice, a 5-bit AO-ADC was achieved, a few years later [11]. Then, replacing the high-nonlinear fiber with a shorter photonic crystal fiber an AO-ADC with the 6-bit resolution was achieved [12]. Recently, a group has employed intensity-towavelength conversion by soliton selffrequency shift and experimentally demonstrated a 40-Gs/s AO-ADC. [13].

Meanwhile, the integrable optical ADC based on a semiconductor optical amplifier (SOA) was first reported in [14]. In this design, the cross-gain modulation of SOA was used to quantize a four-level signal at $20 \mathrm{Gs} / \mathrm{s}$ and code it into 2 bits. However, as the number of the quantization levels $(N)$ is increased for high-resolution applications, this design faces two problematic issues: (i) the complexity of the ADC structure, requiring $2 \mathrm{~N}+1$ SOAs; (ii) the quantization levels becomes hardly distinct. In an alternative design, another research group has exploited polarization switches based on the nonlinear polarization rotation in SOA to achieve a 3-bit ADC operating at hundreds of Gs/s [15]. Later on, using multiple rectangular bandpass filters to digitize the chirped probe signal passing through a quantum dot (QD)-SOA, another research group has revealed a two-bit SOAbased AO-ADC, operating at $10 \mathrm{Gs} / \mathrm{s}$ with an 8-level optical quantization [16].

Furthermore, an alternative method to achieve an integrable all-optical ADC is to exploit the Kerr nonlinearities in a photonic crystal (PhC). In this regard, exploiting two-channel drop filters coupled with a cavity-based wavelength selective reflector, a PhC-based 2 bit ADC with no coder has been proposed [17]. Another group has reported the design of a two-bit PhC-based ADC with a coder, employing a nonlinear demultiplexer to quantize the input analog signal and a coder to convert the quantized levels into a 2-bit binary code [18, 19]. With the aid of an appropriate reflector in conjunction with a hexagonal $\mathrm{PhC}$ waveguide, a 2-bit AO-ADC has been designed without the need for any coder [20]. The authors have shown their design could improve operation speed, without discussing the decrease in its nonlinearities.

In this paper, we are introducing an integrated two-bit optical ADC, consisting of an SOA as a nonlinear optical quantizer integrated with two-channel drop filters devised on a single PhC platform to act as an optical coder. Unlike the fiber-based ADCs, the new design is integrable to $I n P$-based photonic chips and can operate with much less optical input power. Moreover, it is possible to increase its resolution by altering the configuration of the optical coder without a need for an extra SOA, consuming less cross-sectional area and requiring less complicated electronic circuitry. This integrated ADC, unlike the PhC-based ADCs, can operate with no need for exploiting a nonlinear $\mathrm{PhC}$. Besides, the integration of SOA and PhC helps to decrease the ADCs integral nonlinearity (INL) and differential nonlinearity (DNL) errors.

The rest of this paper is organized as follows. Section 2 is devoted to a description of the proposed integrated ADC structure and the basic design principles. In Section 3, we present the simulation results and the related discussions. Finally, we conclude the paper with conclusive remarks in Section 4.

\section{INTEGRATED OPTICAL ADC}

\section{A. Proposed Structure}

Figure 1 illustrates a 3D schematic representation of the proposed integrated 
optical ADC, composed of an InP-based traveling wave (TW) SOA, whose active region is made of $\mathrm{In}_{1-x} \mathrm{Ga}_{x} \mathrm{As}_{y} \mathrm{P}_{1-y}$, integrated with two drop filters devised on an $\operatorname{In} P$ pillar $\mathrm{PhC}$ platform. Details of the SOA and PhC physical and geometrical parameters are given in Section 3.

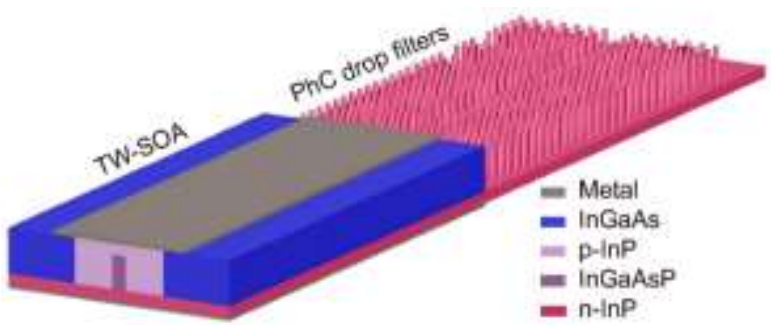

Fig. 1. 3D schematic representation of the proposed integrated optical ADC.

\section{B. Operating principle}

The operating principles of the proposed integrated optical ADC are based on the SPM nonlinear effect in the SOA. Consider a given modulated Gaussian pulse chain as an input optical signal, as schematically illustrated in Fig. 2. The signal at the SOA output, due to the SOA long gain recovery time, exhibits a frequency chirp that depends on the input pulse energy. After passing through the $\mathrm{PhC}$ based drop filters, the chirped signal is quantized into a four-level coded signal.

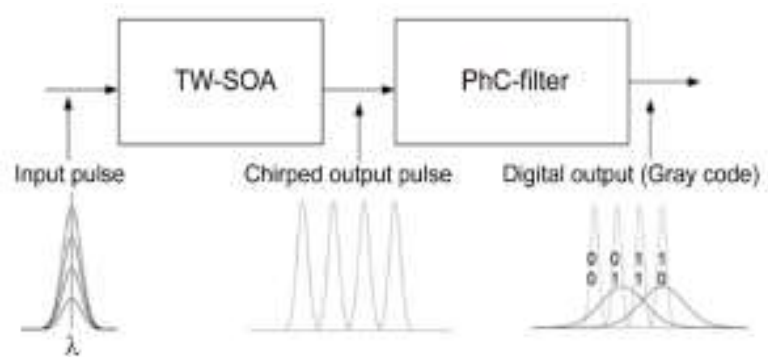

Fig. 2. Schematic of the optical ADC operating principle.

The PhC-based filters are appropriately designed to produce the Gray-code (i.e., 00, 01, 11, and 10). For this purpose, the filter bandwidth determines the pulsewidth of the input pulse. Moreover, to have four distinct output levels, the design is aimed to obtain the maximum possible shift for any given pulsewidth.

\section{SPM-Induced frequency chirp}

The SPM nonlinearity of an SOA, due to its long gain recovery time, is sensitive to the input pulse amplitude. Pulse propagation models in SOA has already been developed by others [20-22]. Employing the model developed by [20], one can describe the evolution of an optical pulse in an SOA by the following three coupled equations:

$\frac{\partial g}{\partial \tau}=\frac{g_{0}-g}{\tau_{c}}-\frac{g P}{E_{\mathrm{sat}}} ; \quad \tau=t-\frac{v_{g}}{z}$

$\frac{\partial P}{\partial z}=\left(g-\alpha_{\text {int }}\right) \cdot P$

$\frac{\partial \varphi}{\partial z}=-\frac{1}{2} \alpha g$

where $z$ and $t$ are the space and time coordinates, $g$, and $P$ and $\varphi$ represent the gain, and the pulse amplitude and phase at position $z$ at any instant $\tau, \tau_{\mathrm{c}}, V_{\mathrm{G}}, \alpha_{\mathrm{int}}$, and $\alpha$ are the spontaneous carrier lifetime, signal group velocity in the active medium, internal loss, and the linewidth enhancement factor. Moreover,

$$
g_{0}=\Gamma a_{N} N_{0}\left(I / I_{0}-1\right)
$$

is the small-signal gain with $\Gamma, a_{\mathrm{N}}, N_{0}$, and $I$, representing the confinement factor, gain coefficient, transparency carrier density, and the injection current. Moreover, for an active layer of length $l$, width $w$, and thickness $d$ the transparency current is $I_{0}=q V N_{0} / \tau_{\mathrm{c}}$ with $q$ and $V=w d l$ being the electron charge and active layer volume. Furthermore,

$$
E_{\mathrm{sat}}=\hbar \omega_{0} \sigma / a_{N}
$$

is the saturation energy, where $\hbar, \omega_{0}$, and $\sigma=w d / \Gamma$ are the reduced Planck's constant, pulse radian frequency, and the mode crosssection. 
Integrating (1a)-(1c) over the SOA length and ignoring the internal loss for the case in which $\alpha_{\text {int }} \ll g$, we get:

$$
\begin{aligned}
& \frac{d \mathcal{G}}{d \tau}=\frac{g_{0} l-\mathcal{G}}{\tau_{c}}-\frac{P_{\text {in }}(\tau)}{E_{\text {sat }}}[\exp (\mathcal{G}-1)] \\
& P_{\text {out }}(\tau)=P_{\text {in }}(\tau) \cdot \exp [\mathcal{G}(\tau)] \\
& \varphi_{\text {out }}(\tau)=\varphi_{\text {in }}(\tau)-\frac{1}{2} \alpha \mathcal{G}(\tau)
\end{aligned}
$$

where $\quad \mathcal{G}(\tau)=\int_{0}^{l} g(z, \tau) d z \quad$ represents the integral gain at any instant $\tau$, on the given temporal Gaussian input pulse of energy $E_{\text {in }}$ and time constant $\tau_{0}$,

$$
P_{\text {in }}(\tau)=\frac{E_{\text {in }}}{\tau_{0} \sqrt{\pi}} \exp \left(-\frac{\tau^{2}}{2 \tau_{0}^{2}}\right) \text {. }
$$

After solving (3a) numerically for $G(\tau)$, one can obtain the output pulse spectrum, by (3b) and its Fourier transform.

Any change in the phase with time corresponds to a chirp in the output signal frequency,

$$
\Delta \omega_{\text {out }} \equiv-\frac{d \varphi_{\text {out }}}{d \tau}=\frac{\alpha}{2} \frac{d \mathcal{G}}{d \tau} .
$$

Using the relation between the wavelength $(\lambda)$ and radian frequency (5) leads to the resulting shift in the output wavelength

$$
\Delta \lambda_{\text {out }}=-\frac{\alpha \lambda^{2}}{2 c} \frac{d \mathcal{G}}{d \tau}
$$

It has already been shown that for an input temporal pulse of width $\Delta \tau_{1 / 2}=2(2 \ln 2)^{1 / 2} \tau_{0}$, the larger the unsaturated single-pass gain of the amplifying cavity (i.e., $G_{0}=\exp \left(g_{0} l\right)$ ) the larger the shift in the wavelength of the chirped signal $\left(\Delta \lambda_{\text {out }}\right)$. Moreover, for an SOA under a certain biasing condition that leads to the maximum $G_{0}$, one may increase the shift in the chirped signal wavelength, using a narrower temporal pulse [20]. However, for the latter case, an increase in $\Delta \lambda_{\text {out }}$ can be achieved at the expense of an increase in the signal frequency or wavelength bandwidth $\left(\Delta \omega_{1 / 2}\right.$ or $\left.\Delta \lambda_{1 / 2}\right)$. Recalling that for a Gaussian temporal pulse of given $\Delta \tau_{1 / 2}$, the wavelength bandwidth is given by $\Delta \lambda_{1 / 2}=-\left(\lambda^{2} / c\right) \Delta \omega_{1 / 2}=$ $-0.44\left(\lambda^{2} / c \Delta \tau_{1 / 2}\right)$, according to the timebandwidth product limitation for an ultra-short Gaussian pulse. Hence, for an appropriately designed $\mathrm{PhC}$ drop filter to be able to code the chirped signals correctly, one needs to choose a Gaussian pulse of suitable width.

\section{PhC Filter}

To provide a coded digital output, the SOA output should be integrated into two appropriately designed $\mathrm{PhC}$ based bandpass filters, with relatively low-quality factors and wide passbands, overlapping over the desired frequency range. Each designed filter is composed of a point defect cavity coupled to a line defect waveguide, similar to that of [17], devised within a pillar $\mathrm{PhC}$ platform on the same InP substrate used for SOA.

\section{III.RESULTS AND DISCUSSION}

The 2D finite difference time domain (FDTD) numerical method is employed to simulate the dynamic behavior of the integrated optical ADC of Fig. 1.

\section{A. Frequency Chirp}

Consider a commercial SOA (CIP Technologies, model SOA-XN-OEC-1550) [25] and employing the constant parameters given in Table 1, solving (2a) and (2b), one gets $E_{\mathrm{sat}}=0.5 \mathrm{pJ}$ and $g_{0}=76.5 \mathrm{~cm}^{-1}\left(G_{0} \approx 33\right.$ $\mathrm{dB})$.

First, using (3)-(6) we calculate the amount of the redshift $\left(\Delta \lambda_{\text {out }}\right)$ versus the normalized pulse energy $\left(E_{\mathrm{in}} / E_{\text {sat }}\right)$ for different temporal pulses of widths 1 ps $\leq \Delta \tau_{1 / 2} \leq 10 \mathrm{ps}$, as illustrated in Fig 3(a). As can be observed from this figure, the redshift, for any given pulsewidth, is a sublinear function of the input pulse energy. Furthermore, for given input energy, the 
shorter the temporal input pulse the larger the redshift experienced by the output signal. Moreover, careful inspection reveals that at any given energy, the SOA SPM incurs a redshift on the output pulse signal that increases linearly with an increase in the input pulse bandwidth $\left(\Delta \lambda_{1 / 2}\right)$. Fig. 3(b) illustrates such linear dependence at four given input energies $\quad\left(E_{\mathrm{in}} / E_{\mathrm{sat}}=0.005,0.01,0.015\right.$, and $0.02)$.

Table 1. SOA Parameters used in simulations [21], [25].

\begin{tabular}{cccc}
\hline \hline Symbol & Quantity & Amount & Unit \\
\hline$l$ & Active region length & 1000 & $\mu \mathrm{m}$ \\
$w$ & Active region width & 0.1 & $\mu \mathrm{m}$ \\
$d$ & Active region thickness & 2.184 & $\mu \mathrm{m}$ \\
$a_{N}$ & Material gain constant & $10.22 \times 10^{-8}$ & $\mu \mathrm{m}^{2}$ \\
$I$ & Injection current & 75 & $\mathrm{~mA}$ \\
$N_{O}$ & Transparency carrier density & $2.7 \times 1023$ & $\mathrm{~m}^{-3}$ \\
$n$ & Active region Modal ref. index & 3.22 & - \\
$\alpha$ & Linewidth enhancement factor & 3 & - \\
$\Gamma$ & Optical confinement factor & 0.47 & - \\
$\lambda$ & wavelength & 1550 & $\mathrm{~nm}$ \\
$\tau c$ & Carrier lifetime & 200 & $\mathrm{ps}$ \\
\hline \hline
\end{tabular}

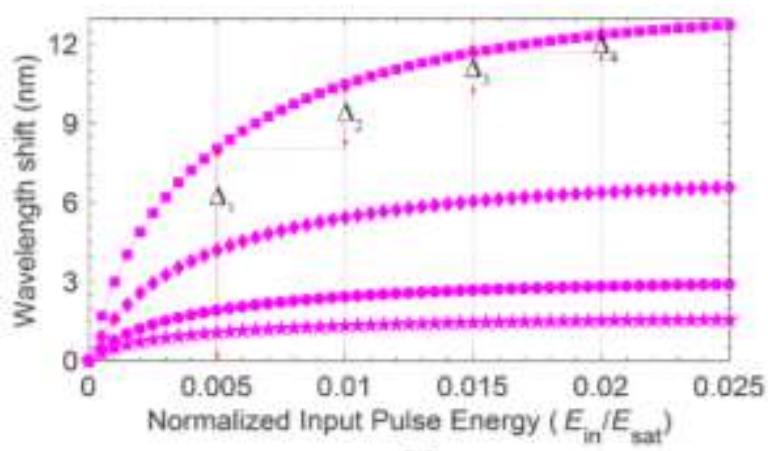

(a)

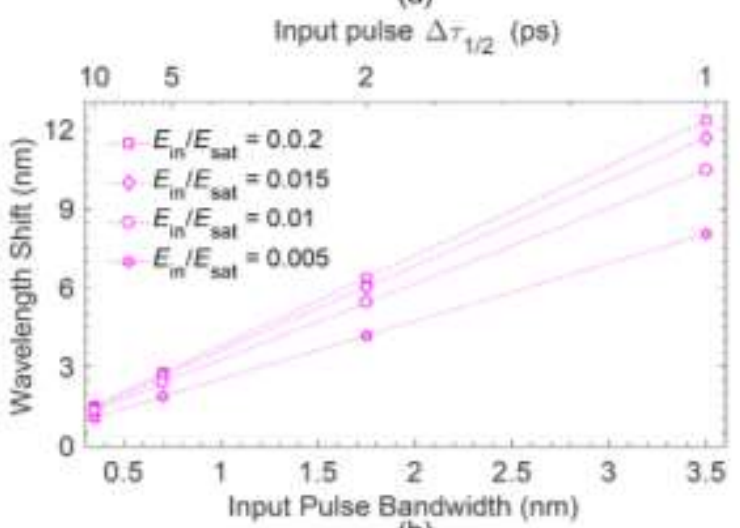

(b)

Fig. 3. The redshift in the signal output wavelength versus (a) the normalized energy of the input pulse $\left(E_{\mathrm{in}} / E_{\mathrm{sar}}\right)$ for various input pulse widths $\left(\Delta \tau_{1 / 2}\right)$; (b) the pulse bandwidth $\left(\Delta \lambda_{1 / 2}\right)$ for various normalized pulse energies.
The slopes of these lines are 2.91, 3.22, 3.25, and 3.39. From this figure, we can conclude that when using the SPM mechanism in an SOA for analog to digital conversion, the appropriate choice of the input Gaussian pulsewidth merely depends on the filter wavelength bandwidth. Because the role of the ratio $\Delta \lambda_{\text {out }} / \Delta \lambda_{1 / 2}$ is more important than the role of the change in the input pulsewidth

Consider the case for $\Delta \tau_{1 / 2}=1$ ps shown by open squares in Fig. 3(a), dividing the input energy range $0 \leq E_{\mathrm{in}} / E_{\mathrm{sat}} \leq 0.02$ into for equal steps $\left(\Delta E_{\mathrm{in}} / E_{\mathrm{sat}}=0.005\right)$, the corresponding redshifts $\left(\Delta_{1,2,3,4}\right)$ can represent an example of four quantization levels of a 4-level quantization scheme shown in Table 2.

Table 2. An example of a four-level quantization scheme for $\Delta \tau_{1 / 2}=1 \mathrm{ps}$.

\begin{tabular}{cccc}
\hline \hline $\begin{array}{c}\text { Range of } \\
\Delta \boldsymbol{E}_{\text {in }}\left(\boldsymbol{E}_{\text {sat }}\right)\end{array}$ & $\begin{array}{c}\Delta \boldsymbol{\lambda}_{\text {out }} \\
(\mathbf{n m})\end{array}$ & $\begin{array}{c}\text { Gray } \\
\text { code }\end{array}$ & $\begin{array}{c}\text { Decimal } \\
\text { value }\end{array}$ \\
\hline $0 \rightarrow 0.005$ & $\Delta_{1}=8.042$ & 00 & 0 \\
$0.1 \rightarrow 0.01$ & $\Delta_{2}=2.438$ & 01 & 1 \\
$0.2 \rightarrow 0.015$ & $\Delta_{3}=1.2$ & 11 & 3 \\
$0.3 \rightarrow 0.02$ & $\Delta_{4}=0.67$ & 10 & 2 \\
\hline \hline
\end{tabular}

From the linear relation between $\Delta \lambda_{\text {out }}$ and $\Delta \lambda_{1 / 2}$ (Fig 3(b)), one can conclude that the ratios $\Delta_{i} / \Delta_{j}(i, j=1,2,3,4)$ should be almost the same for any given input pulsewidth. On the other hand, an ideal ADC produces the digital outputs for equal ranges of the input pulse energy. To have a linear ADC, the bandpass filters should be designed in such a way that they compensate for the nonlinear relation between $\Delta \lambda_{\text {out }}$ and $E_{\text {in }}$ (Fig. 3(a)). Figure 4 illustrates how this compensation can be applied schematically.

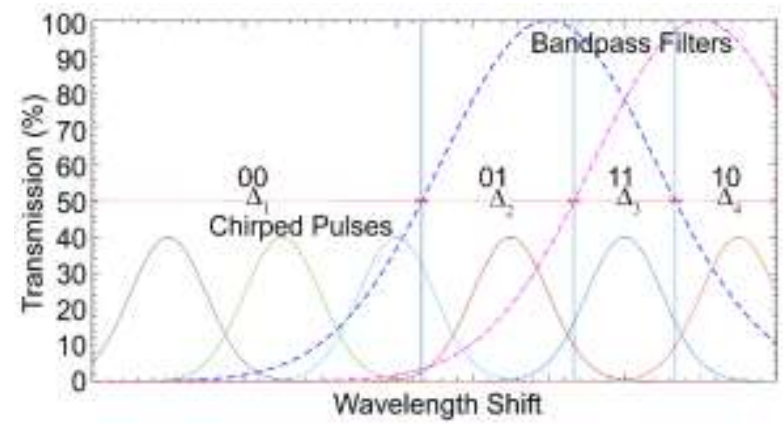

Fig. 4. Optical quantization by $\mathrm{PhC}$ filters. 
The solid curves in the lower parts of the figure represent the output chirped-pulse shifts as the inputs to the bandpass filter and the dashed and the doted-dashed curve represent the bandpass filter output transmission spectra, such that the given ratios between $\Delta_{2,3,4}$ and $\Delta_{1}$ sustain at $50 \%$ transmission, set as the digital bit change $(0 \rightarrow 1)$ recognition level.

\section{B. PhC filter design}

Since the SOA is based on an InP substrate, we consider a cubic $\mathrm{PhC}$ of lattice constant $a$ made by an array of InP pillars of radii $r=0.17 a$. Knowing the relative permittivity of InP at $1550 \mathrm{~nm}$; - i.e., $\varepsilon_{\mathrm{r}} \sim 10$ [23] — we have calculated the $\mathrm{PhC}$ dispersion curves for a transverse magnetic (TM) polarized incident optical beam, employing the plane wave expansion (PWE) method. The results are shown in Fig. 5. As can be observed from this figure, there is a photonic bandgap (PBG) in the normalized frequency range of $0.35 \leq c / a \leq 0.47$. As an example for $a=630 \mathrm{~nm}$, the PBG falls within the wavelength range of $1.3 \mu \mathrm{m}$ to $1.8 \mu \mathrm{m}$.

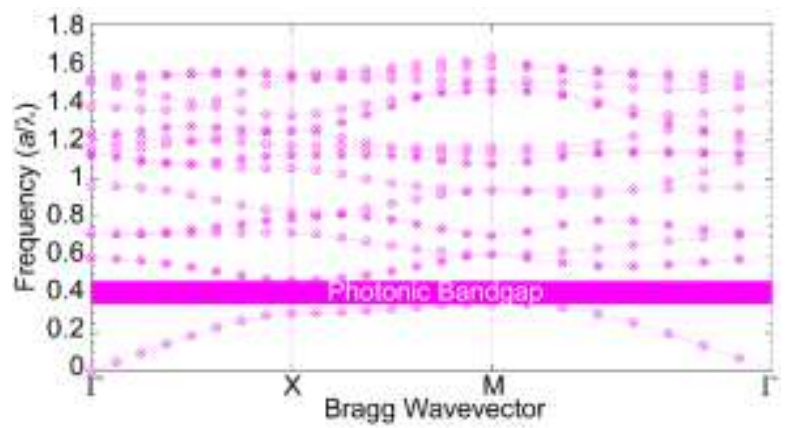

Fig. 5. Dispersion characteristics

Since the drop bandpass filters required for dropping fast and chirped pulses need not be highly selective wavelength filters with wide bandwidths, the point defect $\mathrm{PhC}$ drop filters are good enough. The schematic of a twochannel drop filter with a $W 1$-bus waveguide is shown in Fig. 6.

Considering the radii of the point defects in the outputs 1 and 2 to be $r_{1}=0.1 r$ and $r_{2}=0.112 r$, we have calculated the transmission, using a 1.8 ps TM polarized Gaussian input pulse.

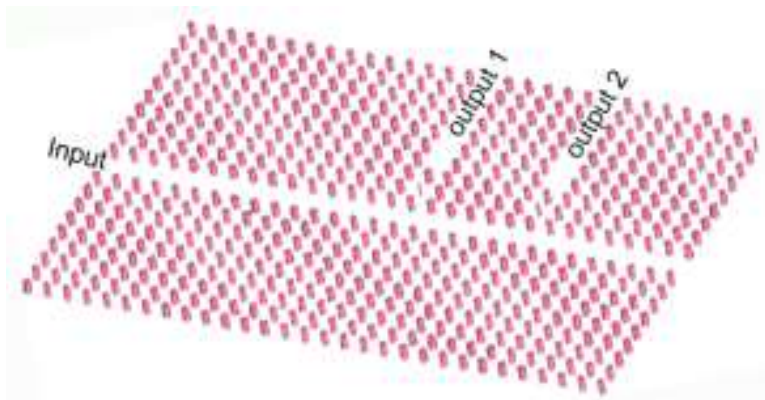

Fig. 6. Top view of the proposed $\mathrm{PhC}$ based twochannel drop filter.

Figure 7 illustrates the transmission spectra from outputs 1 and 2 . The vertical dots at $\lambda=1576.1,1577.6$, and $1578.3 \mathrm{~nm}$ show the boundaries at which one of the two outputs changes its logic state $(0 \rightarrow 1$ or $1 \rightarrow 0)$, considering $T=37.5 \%$ as the boundary between logical 0 and 1 (the threshold for quantization levels).

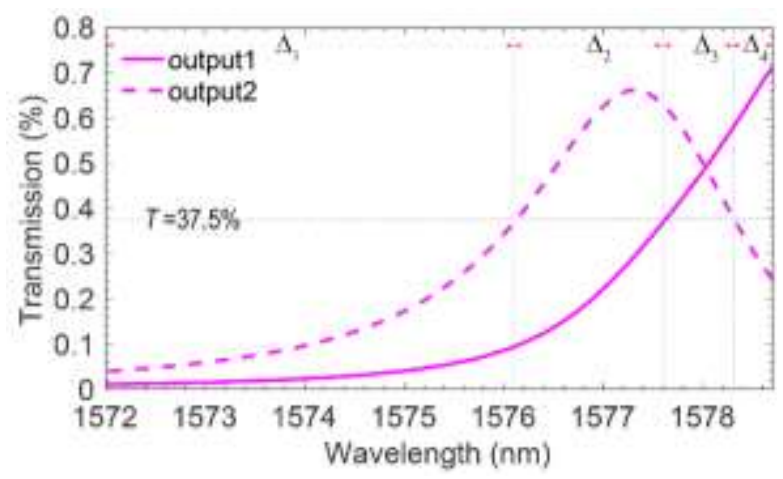

Fig. 7. Transmission spectra emerging from the output 1 (solid squares) and output 2 (open squares) of the drop filter Fig. 6. The horizontal dots at $\mathrm{T}=37.5 \%$ indicates the threshold for the quantization levels.

This figure also shows that the designed filter provides the desired $\Delta_{2} / \Delta_{3}$ ratio. As seen in this figure, $\Delta_{2}$ represents the wavelength range over which output 1 is $\mathrm{ON}(1)$ and output 2 is $\operatorname{OFF}(0)$ whereas $\Delta_{3}$ represents the range over which both outputs are ON. Knowing the values $\Delta_{2}$ and $\Delta_{3}$ and expecting the ratios of $\Delta_{i} / \Delta_{j}$ to be as close as possible to those of Table 2, values of $\Delta_{1}$ and $\Delta_{4}$ can be easily 
determined. Notice, the transmission spectra are depicted only over a wavelength range that equals the sum of the four $\Delta_{i}$ values. According to the value of $\Sigma \Delta_{i} \sim 6.7 \mathrm{~nm}$, this design is capable of properly coding pulses of 2-nm bandwidths (Fig 3.b).

\section{ADC output}

Considering 1.8 ps Gaussian pulses of 1572 nm center wavelengths with different energies launched into the designed ADC input, we have calculated the wavelength shifts experienced by the ADC output, while neglecting the coupling loss between SOA and $\mathrm{PhC}$ filter. The wavelength shift versus the normalized energy is illustrated in Fig. 8.

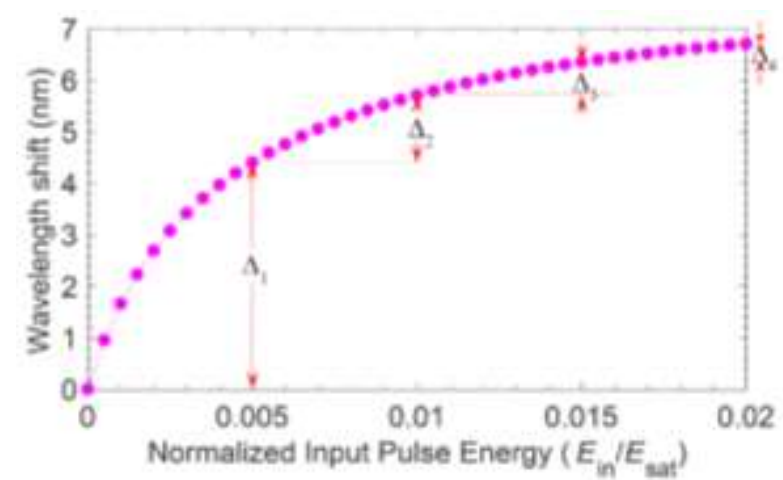

Fig. 8. Wavelength shift exhibited by the ADC output versus the normalized energy of 1.8-ps Gaussian input pulse with $1572 \mathrm{~nm}$ center wavelength.

This figure shows that redshifts obtained for the pulses whose normalized input energies are $E_{\text {in }} / E_{\text {sat }}=0.005,0.01,0.015$, and 0.02 are the desired shifts that we have aimed for.

Launching four chains of 1.8-ps Gaussian pulses with the same center wavelength $(\lambda=1572 \mathrm{~nm})$ but different energies $\left(E_{\mathrm{in}} / E_{\mathrm{sat}}=0.00125,0.00625,0.0125\right.$, and 0.02$)$ four digital state of the ADC output is examined. The simulation results show that the wavelength shifts incurred on the input pulses of given energies, by the SOA, coincide those of Fig. 8, resulting in four different pulses with center wavelengths of $\lambda=1573.95,1576.83$, 1578.08, and $1578.7 \mathrm{~nm}$. Then, the $\mathrm{PhC}$ filter outputs code these four pulses digitally as desired. The time responses of the $\mathrm{PhC}$ filter outputs are illustrated in Fig.9, showing the four digital states $00,01,11$, and 10, considering the $37.5 \%$ transmission level (horizontal dots) as the threshold.

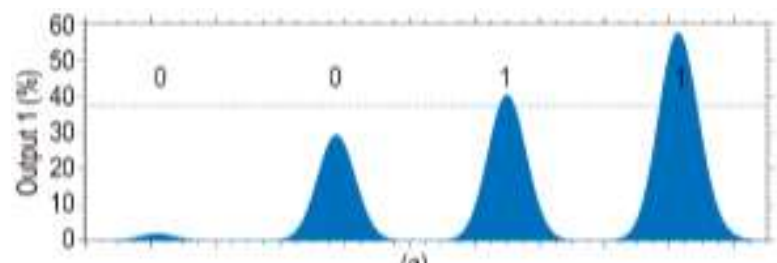

(a)

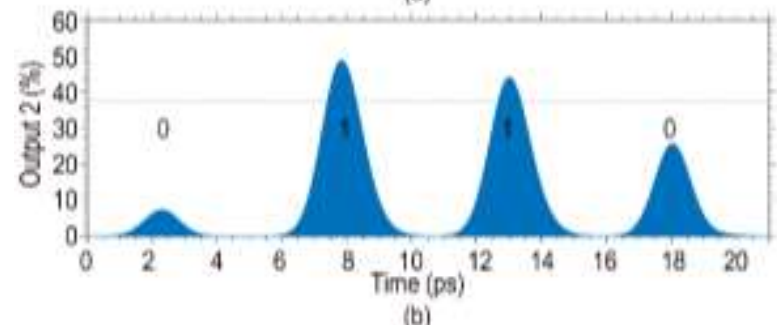

Fig. 9. Time response of the filters (a) output 1 and (b) output 2, depicting the four digital states 00, 01, 11 , and 10 , considering $37.5 \%$ transmission level (horizontal dots) as the threshold.

As can be seen from this figure, the filter response time is shorter than the repetition rate; but the gain recovery time of the SOA is a limiting parameter. Using the data given in Table 1 , the transparency current is $I_{0}=47 \mathrm{~mA}$. The corresponding gain recovery time is found to be $\sim 100$ ps (see Fig. 10). In other words, the proposed AO-ADC sampling rate is $\sim 10 \mathrm{Gs} / \mathrm{s}$.

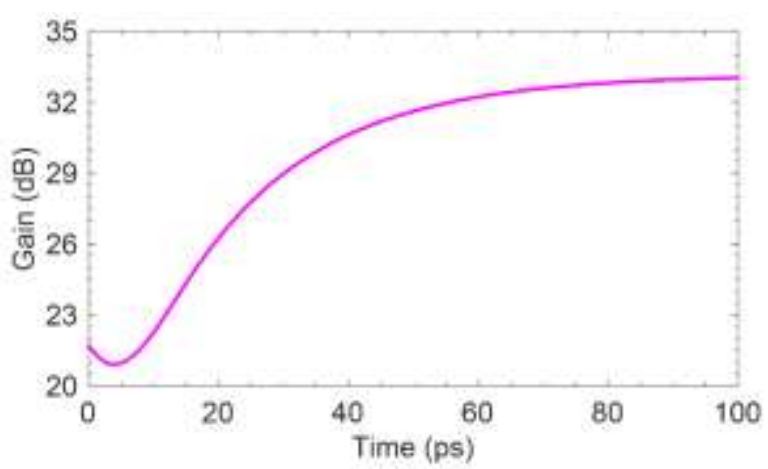

Fig. 10. Gain recovery of the SOA.

The threshold we defined for the filters, the differences between the ratios of $\Delta_{i} / \Delta_{j}$ in the $\mathrm{PhC}$ filters and the SOA wavelength shift are the sources of errors in the proposed ADC. 
These errors make the transfer function of the proposed ADC mismatch with the nominal one. The numerically calculated ADC transfer function is depicted in Fig. 11 and compared with the ideal one.

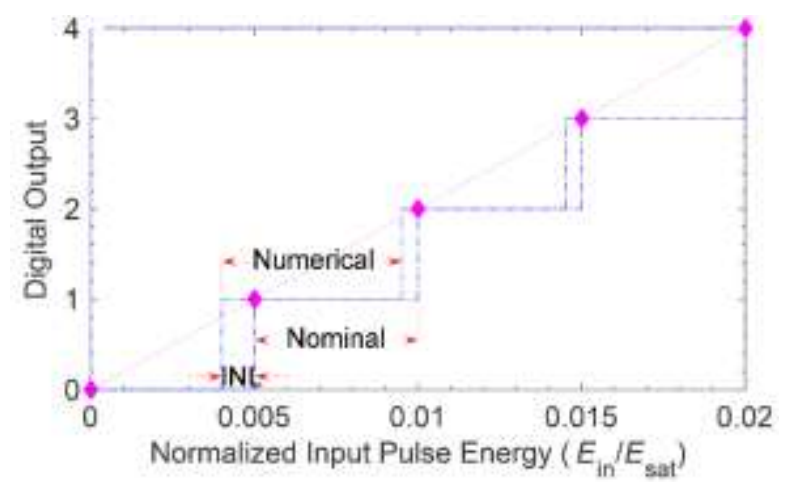

Fig. 11. ADC transfer function.

From this figure, one can estimate the INL (the difference between the nominal and numerically calculated transfer functions) and the DNL errors, corresponding to each digital state. The INL and DNL errors in terms of the least significant bit (LSB), corresponding to four digital states are shown in Fig. 12. The maxima for DNL and INL errors are the same (0.2 LSB), corresponding to states zero and one, respectively.

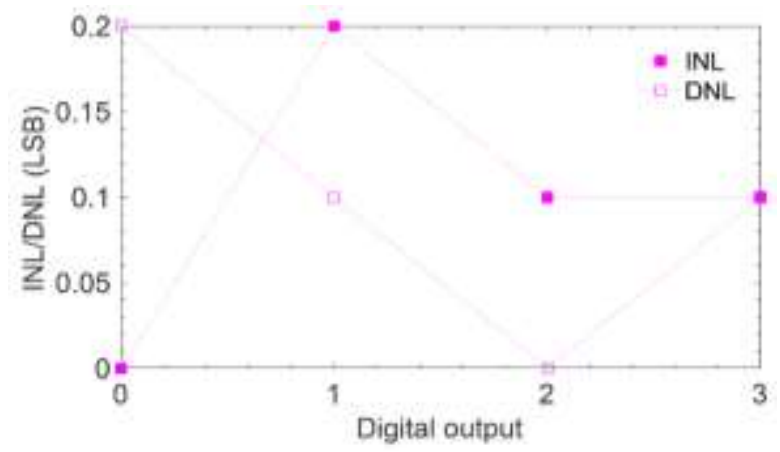

Fig. 12. INL (solid squares) and DNL (open squares) errors versus the digital output.

Finally, we have compared the parameters obtained for the proposed ADC with other optical ADC structures reported in the past decade, as tabulated in Table 3.
Among these, the SOA and PhC-based ADCs have the ability of integration, while the Fiberbased generate more quantization levels (QL).

Table 3. Comparison of parameters for various Optical ADCs

\begin{tabular}{|c|c|c|c|c|c|c|}
\hline $\mathrm{ADC}$ & $\begin{array}{l}\text { Speed } \\
(\mathrm{Gs} / \mathrm{s})\end{array}$ & $\begin{array}{l}\mathrm{QL} \\
\text { (bit) }\end{array}$ & $\begin{array}{l}\text { Power } \\
(\mathrm{mW})\end{array}$ & $\begin{array}{c}\text { INL } \\
(\mathrm{LSB})\end{array}$ & $\begin{array}{c}\text { DNL } \\
\text { (LSB) }\end{array}$ & Ref \\
\hline HNLF & 40 & 64 & N/A & N/A & N/A & [6] \\
\hline HNLF & N/A & 3 & 10.5 & N/A & N/A & [7] \\
\hline $\mathrm{DFF}^{\mathrm{b}}$ & 40 & 4 & 2560 & N/A & N/A & [8] \\
\hline $\begin{array}{c}\mathrm{ADF} / \mathrm{Z} \\
\mathrm{DF}^{\mathrm{c}}\end{array}$ & 160 & 4 & $\begin{array}{c}1200 / 60 \\
0\end{array}$ & N/A & N/A & [9] \\
\hline HNLF & 100 & 16 & 33500 & N/A & N/A & [10] \\
\hline HNLF & 40 & 32 & 45 & 0.875 & 0.75 & [11] \\
\hline $\mathrm{PCF}^{\mathrm{d}}$ & N/A & 6 & 375 & N/A & N/A & [12] \\
\hline HNLF & 40 & 16 & 200 & 0.4 & 0.5 & [13] \\
\hline SOA & 20 & 4 & 8 & N/A & N/A & [14] \\
\hline SOA & $\times 100$ & 8 & 4.8 & 0.7 & 0.4 & [15] \\
\hline $\begin{array}{l}\text { QD- } \\
\text { SOA }\end{array}$ & 10 & 8 & 10.5 & 0.5 & 0.7 & [16] \\
\hline $\mathrm{PhC}$ & 45 & 5 & $60 / \mu \mathrm{m}^{2}$ & N/A & N/A & [17] \\
\hline $\mathrm{PhC}$ & 200 & 4 & $16 / \mathrm{mm}^{2}$ & N/A & N/A & [18] \\
\hline $\mathrm{PhC}$ & 52 & 4 & $100 / \mu \mathrm{m}^{2}$ & N/A & N/A & [19] \\
\hline $\mathrm{PhC}$ & 1000 & 4 & $>25$ & N/A & N/A & [20] \\
\hline $\begin{array}{l}\text { SOA- } \\
\mathrm{PhC}\end{array}$ & 10 & 4 & 4.8 & 0.2 & 0.2 & Present. \\
\hline
\end{tabular}

The power reported in this table is the input analog signal power and does not relate to what the ADC structure consumes. The PhC based ADCs do not need any power supplies and operate in the presence of input signals, while SOA-based ADCs power consumption depends on the numbers of SOA used. For example, in [14] and [16], five and four SOAs are used to design 2-bit ADCs respectively, while in the present work a single SOA used that indicates this design consumes less overall power. Nonetheless, the input power for the present $\mathrm{ADC}$ is the lowest among all SOA based ADCs.

\section{IV.CONCLUSION}

Integrating an InGaAsP SOA with two pointdefect drop filters devised on an InP pillar PhC platform, we have designed an integrated SOA-PhC AO-ADC that can convert 1.8-ps amplitude modulated Gaussian pulses of center wavelengths $1572 \mathrm{~nm}$ and energies $\leq 10 \mathrm{fJ}$ into four coded digital output levels. The self-phase modulation effect in the appropriately designed SOA, due to its long gain recovery time, induce frequency chirps, depending on the pulse energy, at the leading and trailing edges 
of the signal passing through it. The two bandpass $\mathrm{PhC}$ filters are appropriately designed to digitize and code the shifted signals, leading to the Gray code $(00,01,11$, and 10). Designing appropriate filters according to the range of redshifts, we have obtained the key element of this structure to be as linear as it can. The proposed integrated AO-ADC can be used in a photonic network like an all-optical quaternary ASK to OOK modulation format converter or an all-optical BEM system. The proposed design can be developed to have more quantization levels as a new generation of integrated AO-ADCs.

\section{REFERENCES}

[1] H. F. Taylor, H. F. M. J. Taylor, and P. W. Bauer, "Electro-optic analog-to-digital conversion using channel waveguide modulators," Appl. Phys. Lett. Vol. 32, pp. 559-561, 1978.

[2] L. Y. Nathawad, R. Urata, B. A. Wooley, and D. A. B. Miller, "A 40-GHz-bandwidth, 4-bit, time-interleaved A/D converter using photoconductive sampling," IEEE J. SolidState Circuits, Vol. 38, pp. 2021-2030, 2003.

[3] S. Yang, C. Wang, H. Chi, X. Zhang, S. Zheng, X. Jin, and J. Yao, "Photonic analog-to-digital converter using MachZehnder modulators having identical halfwave voltages with improved bit resolution," Appl. Opt. Vol. 48, pp. 4458-4467, 2009.

[4] J.-M. Jeong and M. E. Marhic, "All-optical analog-to-digital conversion implemented by a nonlinear fiber interferometer," Opt. Commun. Vol. 91, pp. 115-122, 1992.

[5] L. Brozozowski and E. H. Sargent, "Alloptical analog-to-digital converters, hard limiters, and logic gates," J. Lightw. Technol. Vol. 19, pp. 114-119, 2001.

[6] Ch. $\mathrm{Xu}$ and $\mathrm{Xi}$. Liu, "Photonic analog-todigital converter using Soliton self-frequency shift and interleaving spectral filters," Opt. Lett. Vol. 28, pp. 986-988, 2003.

[7] T. Nishitani, T. Konishi, and K. Itoh, "Integration of proposed all-optical analog-todigital converter using self-frequency shifting in fiber and a pulse-shaping technique," Opt. Rev. Vol. 12, pp. 237-241, 2005.

[8] Sh-i. Oda and A. Maruta, "A novel quantization scheme by slicing supercontinuum spectrum for all-optical analog-to-digital conversion," IEEE Photon. Technol. Lett. Vol. 17, pp. 465-467, 2005.

[9] Sh. Oda and A. Maruta, "Two-bit all-optical analog-to-digital conversion by filtering broadened and split spectrum induced by Soliton effects on self-phase modulation in fiber," IEEE J. Sel. Top. Quantum Electron. Vol. 12, pp. 307-314, 2006.

[10]T. Nishitani, T. Konishi, and K. Itoh, "Resolution improvement of all-optical analog-to-digital conversion employing selffrequency shift and self-phase-modulation induced spectral compression," IEEE J. Sel. Top. Quantum Electron. Vol. 14, pp. 724-732, 2008.

[11]T. Konishi, K. Takahashi, H. Matsui, T. Satoh, and K. Itoh, "Five-bit parallel operation of optical quantization and coding for photonic analog-to-digital conversion," Opt. Exp. Vol. 19, pp. 16106-16114, 2011.

[12] K. Zhe, Y. Jin-Hui, L. Sha, X. Song-Lin, Y. Bin-Bin, S. Xin-Zhu, and Y. Chong-Xiu, "Sixbit all optical quantization using photonic crystal fiber with pre-chirped spectral compression techniques," Chin. Phys. Vol. B 22, pp. 114211 (1-5), 2013.

[13] T. Nagashima, M. Hasegawa, and T. Konishi, "40GSampl/s all-optical analog to digital with resolution degradation prevention," IEEE Photon. Technol. Lett. Vol. 29, pp. 74-77, 2017.

[14] M. Scaffardi, E, Lazzeri, F. Fresi, L. Poti L, and A. Bogoni. "Analog-to-Digital Conversion based on Modular Blocks Exploiting Cross-gain Modulation in Semiconductor Amplifiers," IEEE Photon. Technol. Lett. Vol. 21, pp. 540-542, 2009.

[15]H. Wen, H. Wang, and Y. Ji, "All-optical quantization and coding scheme for ultra-fast analog-to-digital conversion exploiting polarization switches based on nonlinear polarization rotation in semiconductor optical amplifiers," Opt. Commun. Vol. 285, pp. 3877-3885, 2012. 
[16] H. Hoshino, T. Okada, and M. Matsuura, "Photonic analog-to-digital conversion using a red frequency chirp in a semiconductor optical amplifier," Opt. Lett. Vol. 43, pp. 2272-2275, 2018.

[17]B. Yousefi, M. K. Moravvej-Farshi, and N. Granpayeh, "Two-bit all-optical analog-todigital converter based on nonlinear Kerr effect in 2D photonic crystals," Opt. Commun. Vol. 285, pp. 3228-3223, 2012.

[18]F. Mehdizadeh, M. Soroosh, H. AlipourBanaei, and E. Farshidi, "All Optical 2-bit Analog to Digital Converter Using Photonic Crystal Based Cavities," Opt. Quantum Electron. Vol. 49, pp. 1-11, 2017.

[19]F. Mehdizadeh, M. Soroosh, H. AlipourBanaei, and E. Farshidi, "A novel proposal for all optical analog-to-digital converter based on photonic crystal structures," IEEE Photon. J. Vol. 9, pp. 4700311 (1-12), 2017.

[20] D. Jafari, T. Nurmohammadi, M. J. Asadi, and K. Abbasian "All-Optical Analog-to-Digital Converter based on Kerr Effect in Photonic Crystal," Opt. Laser Technol. Vol. 101, pp. 138-143, 2018.

[21] G. P. Agrawal and N. A. Olsson, "Self-phase modulation and spectral broadening of optical pulses in semiconductor laser amplifiers," IEEE J. Quantum Electron. Vol. 25, pp. 2297 2306, 1989.

[22] P. P. Baveja, D. N. Maywar, A. M. Kaplan, and G. P. Agrawal, "Spectral broadening in ultrafast semiconductor optical amplifiers induced by gain dynamics and self-phase modulation," Opt. Lett. Vol. 35, 294-296 2010.

[23] M. Razaghi, V. Ahmadi, and M. J. Connely, "Comprehensive finite-difference timedependent beam propagation model of counterpropagating picosecond pulses in a semiconductor optical amplifier," J. Lightw. Technol. Vol. 27, pp. 3162-3174, 2009.

[24]M. J. Connely, "Wideband semiconductor optical amplifier steady-state numerical model," IEEE J. Quantum Electron. Vol. 37, pp. 349-447, 2001.

[25] P. Baveja, D. N. Maywar, and G. P. Agrawal. "Interband Four-Wave Mixing in Semiconductor Optical Amplifiers with ASEEnhanced Gain Recovery," IEEE J. Selec.
Topics Quantum Electron. Vol. 18, pp. 899908, 2012.

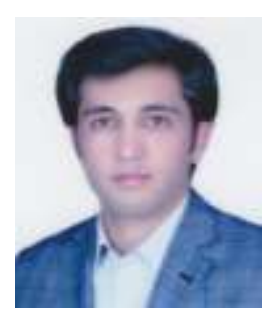

Sajjad Moshfe is currently a Ph.D. candidate, working on his dissertation at the Department of Electrical Engineering, Science and research branch, Islamic Azad University, Tehran, Iran.

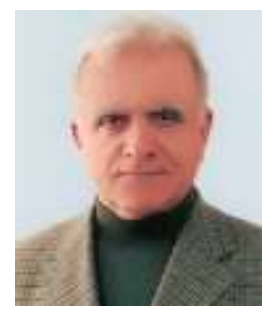

M. Kazem Moravvej-Farshi received the B.Sc. and the M.A. degrees in physics from Sharif University of Technology (SUT), Tehran, Iran, in 1976, and the University of Southern California (USC), Los Angeles, California, in 1978, respectively, the M.Sc. and the Ph.D degrees in electronics from the University of California at Santa Barbara (UCSB), in 1980, and the University of New South Wales (UNSW), Sydney, Australia, in 1987, respectively. From 1980 to 1984, he was a member of research staff with the Division of Microwave, Iran Telecommunication Research Center (ITRC). He joined Tarbiat Modares University (TMU) in 1987, where he is a Professor of Electronics and the head of the Nano Plasmo-Photonics research group. Professor MK Moravvej-Farshi was elected as one of the two most prominent professors of 2002 in the field of electrical engineering, nationwide.

Professor Moravvej Farshi is one of the founding members of the Optics and Photonics Society of Iran (OPSI) and is currently serving as the president of OPSI (2016-2019). He is a Senior Member of the Optical Society of 
America (OSA) and also IEEE Photonics and Electron Device Societies.

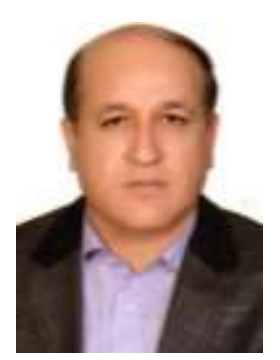

Kambiz Abedi was born in Ahar, Iran, in 1970. He received the B.Sc. degree from the
University of Tehran, Tehran, Iran, in 1992, the M.Sc. degree from Iran University of Science and Technology, Tehran, in 1995, and the Ph.D. degree from Tarbiat Modares University, Tehran, in 2008, all in electrical engineering. $\mathrm{He}$ is currently an Associate Professor at Shahid Beheshti University, Tehran, Iran. His current research interests include design, circuit modeling, and numerical simulation of optoelectronic devices, semiconductor lasers, optical modulators, optical amplifiers, detectors, plasmonic-photonic devices, MOEMS devices, slow light, and photonic crystals. 
THIS PAGE IS INTENTIONALLY LEFT BLANK. 